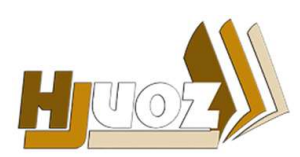

hjuoz.uoz.edu.krd p-ISSN: $2410-7557$ e-ISSN: 2518-5128

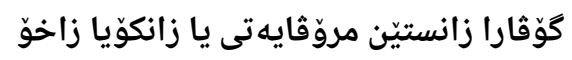

مجلة العلوم الانسانية لجامعة زاخو مروفئ

Humanities Journal of University of Zakho (HJUOZ)

Vol. 6, No. 1,Part 2, pp. 280-289, Mar-2018

\title{
دود الإدارة الإستراتيجية في تحقيق التفوق التسويقي في المنظمات الصناعية
}

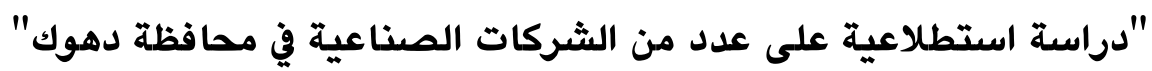

\author{
خضر خليل شيخو الجرجري
}

المعهد التقني - زاخو، قسم إدارة المستشفيات، جامعة دهوك التقنية، إقليم كوردستان -العراق. (khder_76@yahoo.com)

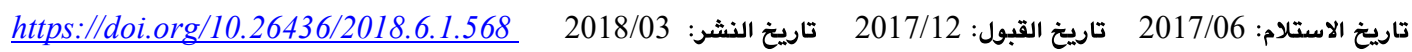

الملخص:

تهدف الشركات الصناعية الى تحقيق أهدافها ولعل أهم هدف تسعى الى تدقيقها هو التفوق التسويقي على المنافسين، من أجل البقاء والنمو في دنيا الأعمال، وتعتبر وجود الإدارة الاستراتيجية في الشركات الصناعية إحدى أهم العوامل التي يمكن من خلالها الوصول الى هدف التفوق التسويقي، لما لها من دود فعال في توظيف امكانيات الشركة والتكييف مع المتغييرات الداخلية والخارجية. وقد اختيرت مجموعة مكونة من خمسة شركات صناعية لتكون مجالاً للتطبيق الميداني للبحث، واعتمد الباحث أسلوب الاستبانة بوصفه أداة رئيسة لجمع البيانات والمعلومات في الجانب العملي للبحث، ومن أجل تحقيق أهداف البحث وفرضياتها تم وضع نموذج افتراضي يوضح طبيعة العلاقة بين المتغيرات المستقلة والمعتمدة للبحث. ويناءاً على وصف متغيرات البحث وتشخيصها واختبار علاقات الارتباط والتأثير تم التوصل الى مجموعة من الاستنتاجات والتي أكدت على وجود علاقة ارتباط وأثر معنوي بين الادارة الاستراتيجية والتفوق التسويقي للشركات المبحوثة. وفي ضوء الاستنتاجات التي توصل إليها تقدم الباحث بعدد من المقترحات من أبرزها ضرورة

تفعيل دود الادارة العليا في تبني الإدارة الاستراتيجية بما يعزن تحقيق التفوق التسويقي للشركات المبحوثة. الكلمات الدالة: الادارة الاستراتيجية، مراحل الادارة الاستراتيجية، التفوق التسويقي، رضا الزبائن، ولاء الزبائن.

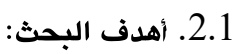

تكمن أهدف البحث من خلال الآتي:

1. توضيح مفهوم الإدارة الإستراتيجية.

2. توضيح التفوق التسويقي وابعادهُ المختلفة.

3. بيان دور الإدارة الإستراتيجية في تحقيق التفوق التسويقي.

3.1

يتجلى أهمية البحث من خلال النقاط الآتية:

1. ابراز دود الإدارة الإستراتيجية في استشراف المستقبل للشركات

المبحوثة.

2. بيان أهمية الإدارة الإستراتيجية في مواجهة التحديات الداخلية

$$
\text { والخارجية للشركات المبحوثة. }
$$

3. بيان أهمية التفوق التسويقي للشركات المبحوثة.

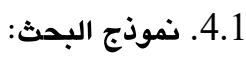

تتطلب المعالجة المنهجية لمشكلة البحث في ضوء اطارما النظري ومضامينه الميدانية تصميم نموذج افتراضي كما في الشكل(1) والذي يشير الى علاقات الإرتباط والتاثير بين الإدارة الإستراتيجية

$$
\text { والتفوق التسويقي للشركات المبحوث. }
$$

\section{1.منهجية البحث}

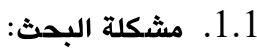

تكمن مشكلة البحث في قلة امتمام الشركات في القطاع الصناعي بالدود الإستراتيجي في التكيف مع البيئة الداخلية والخارجية

للشركات، ولهذا يحدث قصور وضعف في مجال تسويق منتجاتها. ومن خلال ما سبق يمكن طرح إثكالية مفادما: كيف يمكن أن يساهم تطبيق الإدارة الإستراتيجية في تصسين التفوق التسويقي

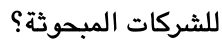
ومن أجل معالجة أكثر دقة، يتعين طرح مجموعة من التساؤلات الفرعية: 1. مل منالك تطبيق فعلي للإدارة الإستراتيجية في الشركات

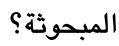

2. ماهي أسباب ضعف التفوق التسويقي في الشركات المبحوثة ؟ 3. مل بتطبيق الإدارة الاستراتيجية في الشركات المبحوثة يتحقق تفوق تسويقي لها؟ 


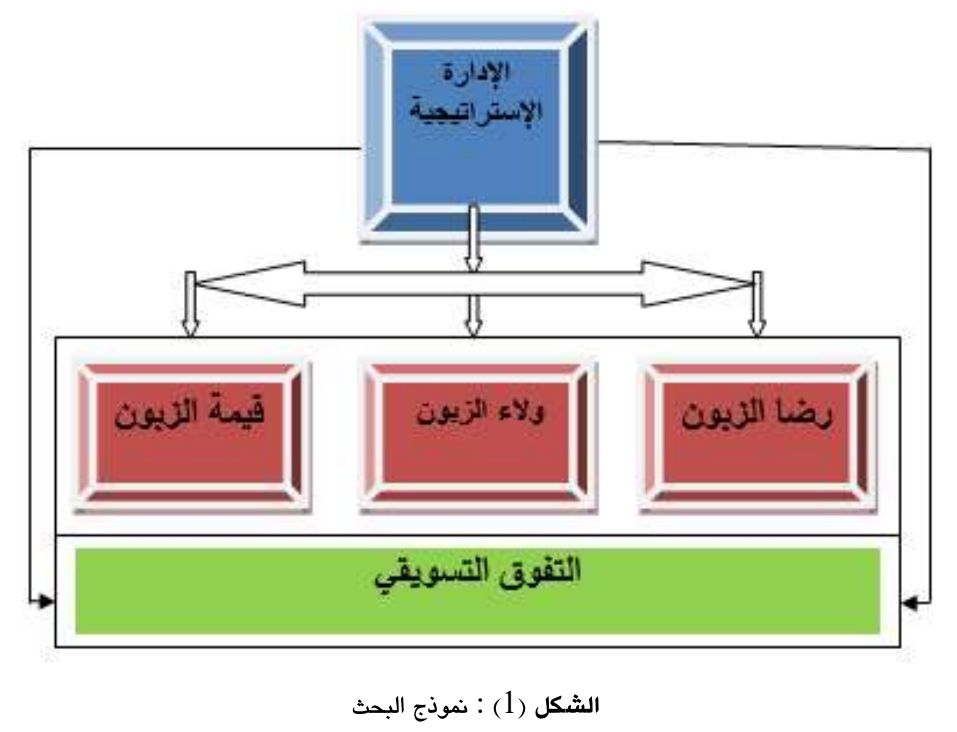

اللازمة لهذه الأهداف، ولذلك فإن الاستراتيجية تجمع بين صياغة 5.1

الأهداف البشرية والتنظيم من النشاط البشري لتحقيق تلك الأهداف

.(White, 2004, 5)

ومن وجهة نظر ( David , 2011, 6 ) يشير الى أنَّ الإدارة الاستراتيجية هي فن وعلم صياغة، وتنفيذ، وتقييم القرارات متعددة الوظائف التي تمكن المنظمة من تحقيق أهدافها. وتشمل تركيز الإدارة الاستراتيجية على دمج إدارة التسويق، والتمويل، والإنتاج، والبحث والتطوير، ونظم المعلومات، لتحقيق النجاح التنظيمي. ويستخدم مصطلح الإدارة الاستراتيجية للإثارة إلى صياغة الاستراتيجية وتنفيذها، والتقييم، والغرض منها هي استغلال وخلق فرص جديدة

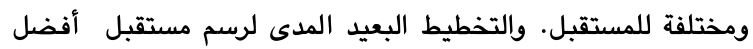
من خلال محاولة تحسين الاتجامات الحالية.

2.2. أهمية الإدارة الإستراتيجية:

تنبع أهمية الإدارة الإستراتيجية في كونها تساعد الشركات التي تتبناها في التنبؤ بمستقبلها، ومحاوله رسم الخارطة المستقبلية لها والسير عليها، كما تساهم بشكل فعال في السعي لتطوير الشركة باستمرار، من أجل مواكبة آخر التقنيات المستخدمة في عمليات الانتاج ويالتالي منح الشركة فرصة أكبر لمنافسة الشركات المحلية والدولية، محققة بذلك تفوق تسويقي يضمن لها النمو والبقاء في دنيا

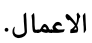
ويشير (تبيدي، 2010، 30) أنَّ أهمية الإدارة الإستراتيجية في الشركات تتمثل في تحقيق الآتي: 1. جعل المديرين أكثر استجابةً وعوعياً لما يدود في البيئة الداخلية

والخارجية. 2. تؤدي الى تنمية وتطوير أساليب التفكير في المستقبل. 3. تعمل على دقة التنبؤ بنتائج التصرفات الإستراتيجية. 4. تسامم في تحسين الاداء العام للشركة على المدى البعيد.
لغرض استكمال متطلبات البحث في إطارهِ العملي وفي ضوء مشكلة البحث وأهدافه واختبار طبيعة العلاقة القائمة بين المتغيرات الرئيسة إستهات والفرعية يتطلب وضع نموذج للبحث يوضح حدود وطبيعة تلك المتغيرات والعلاقات بينها، كما موضح في الشكل (1). واستناداً لنموذج البحث ومشكلته وأمدافه ثم صياغة مجموعة فرضيات إحصائية يتطلب اختبارها وهي:

1.5.1. الفرضية الرئيسة الأولى: توجد علاقة ارتباط معنوية موجبة بين الإدارة الإستراتيجية وتحقيق التفوق التسويقي في الشركات

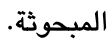

ويتفرع من مذه الفرضية الرئيسة الفرضيات الفرعية والمتمثلة بوجود علاقة إرتباط بين الإدارة الإستراتيجية مع كل بُعد من أبعاد التفوق

$$
\text { التسويقي في الشركات المبحوثة. }
$$

2.5.1. الرئيسة الثانية: هناك تأثير معنوي للإدارة الإستراتيجية

$$
\text { في تحقيق التفوق التسويقي في الشركات المبحوثة. }
$$

ويتفرع من هذه الفرضية الرئيسة الفرضيات الفرعية والمتمثلة بوجود تاثير بين الإدارة الإستراتيجية مع كل بُعد من أبعاد التفوق التسويقي

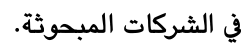

\section{2. الإدارة الإستراتيجية}

1.2. مفهوم الإدارة الإستراتيجية: الادارة الاستراتيجية مي منظومة من العمليات المتكاملة، ذات العلاقة بتحليل البيئة الداخلية والخارجية، وصياغة استراتيجيات مناسبة،

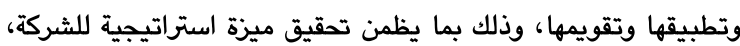

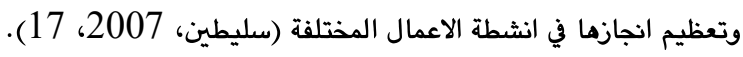
وتمثل الادارة الاستراتيجية كذلك تحديد الأهداف الأساسية، طويلة الأجل وأهداف الشركة، واعتماد مسارات العمل والتوزيع من الموارد 
في حين يشير كل من ,Sepehri \& Kermanshah, 2012

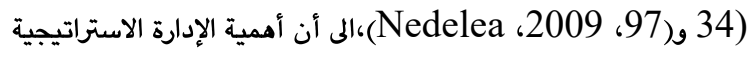
تكمن أن لديها خمس مهام حاسمة لنجاح الشركة، كما مبين في الشكل(2)الآتي:
5. توحيد وتنسيق جهود جميع الإدارات لتحقيق أهداف وغايات محدة. - مدر 6. تساهم في تعديد توجهات الشركة المستقبلية. 7. تساعد في تحديد وتقدير الفرص المستقبلية والمشكلات المتوقعة.

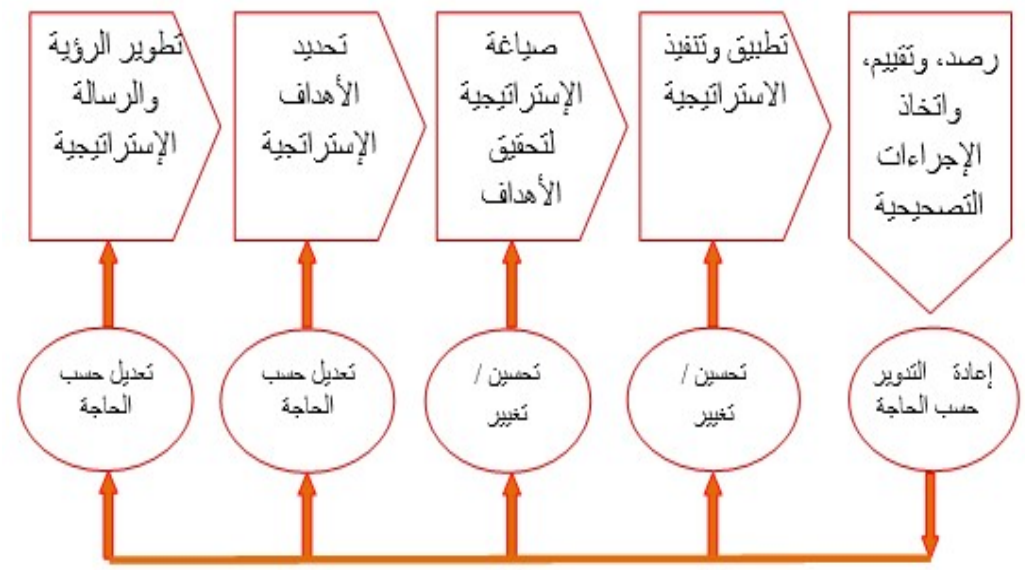

شكل (2): مهام الإدارة الإستراتيجية

Sources: (Nedelea, Stefan ,2009) and (Sepehri, et al. 2012).

ونقاط القوة والضعف بأسلوب فعال، وتتضمن عملية الصياغة الإستراتيجية التحديد الدقيق لكل من الحالات الآتية: (السليماني،

$$
\text { (30، 2012 }
$$

1. تصديد رسالة المنظمة. 2. تحديد الأهداف القابلة للتحقيق. 3. وضع الإستراتيجيات وتطويرها.

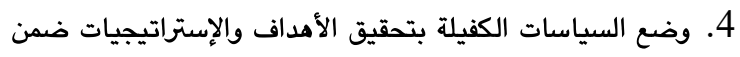
إطار رسالة المنظمة. 3.3.2. مرحلة تنفيذ الإستراتيجية:التفيذ الإستراتيجي هو إيصال وتفسير الخيار الإستراتيجي وضوضعه موضع التطبيق من خلال تحديد المتطلبات التنظيمية والموارد المادية والبشرية اللازمة وممارسة الوظائف الإدارية لتحقيق أهداف الشركة، ويعبارة اخرى هي مجموعة النشاطات والفعاليات التي تمارس لوضع الخطط والسياسات في التنفيذ من خلال البرامج التنفيذية والميزانيات والإجراءات

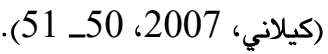

4.3.2.الرقابة الاستراتيجية: تشير (سليطين، 2007، 89) الى أنَّ الرقابة الاستراتيجية هي عملية مستمرة تأخذ مكانها على كل مستويات المنظمة، ويقوم بها المدراء الاستراتيجيون في المنظمة، وتهدف الى تقويم ما تَّ تخطيطهُ، ومنع وقوع الخطأ قبل حدوثهُ، وتقويم العملية التنفيذية (الجارية والاحقة) وذلك لجعل أداء المنظمة في أحسن الحالات. وللرقابة الاستراتيجية أهمية كبيرة للشركة تتمثل في
3.2. مراحل الإدارة الإستراتيجية يمر الإدارة الإستراتيجية باربعة مراحل، وكالآتي: 1.3.2. مرحلة التصميم: ويطلق عليها أيضاً مرحلة التخطيط الإستراتيجي أو الصياغة، وتهتم مرحلة التصميم بوضع الرؤية والرسالة، وتقييم تحليل البيئة الداخلية والخارجية وتحديد الفجوة الإستراتيجية وضع أهداف طويلة الأجل واختيار أفضل الاستراتيجيات الكلية واستراتيجيات الوحدات الإستراتيجية والاستراتيجيات الوظيفية. ويلاحظ أنَّ رسالة المنظمة تحدد البيئة التي سوف يتم تجميع المعلومات عنها، وفي نفس الوقت فانَّ اكتشاف نقاط القوة والضعف والفرص والتهديدات من خلال عملية التغير يمكن أن تؤدى إلى تغيير رسالة المنظمة وتتطلب عملية التصميم تجميع المعلومات وتحليلها واتخاذ قرارات اختيار أفضل البدائل في كل خطوة من خطواتها وينبغي أن تمارس بأعلى درجة من الكفاءة حيث أنَّ نتائجها ذات أثر طويل الأجل يحد لفترة طويلة نوع النشاط الذي تركز عليه المنظمة وما تقدمه من خدمات وسلع والأسواق التي تخدمها والتكنولوجيا المستخدمة والبحوث التي سوف تجرى والموارد

$$
\text { التي سوف تستخدم. (تبيدي ، 2010، 34) }
$$

2.3.2. صياغة الإستراتيجية: يقصد بصياغة الإستراتيجية وضع خطط طويلة الأمد لتمكن الإدارة العليا من إدارة الفرص والتهديدات 
جذب انتباه المختصين بشكل كبير في البحوث والدراسات الأكاديمية مثل رضا الزبون، لأنَّ رضا الزبون يعتبر أحد الأصول التسويقية

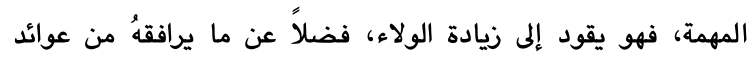

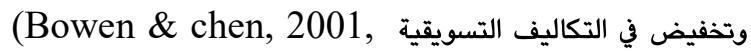
(213) حول المنتجات المقدمة لهُ من داخل الشركة وخارجها (العفيشات، .56، 2001 ويشير (172, Kotler ,2006 ) بأنَّ رضا الزبون هو الإحساس الذي يولد الإنطباع الإيجابي أو السلبي المدرك من طرف الزبون عند مقارنته الفائدة الفعلية من الخدمة أو المنتج مع توقعاته. وعليه يعتمد له رضا الزبون على أداء المنتج نسبة الى توقعات المشتري، وأنَّ الزيون يواجه درجات مختلفة من الرضا، فاذا طابق أداء المنتج لتوقعاته

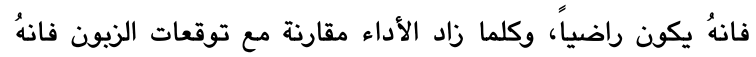
سيحصل على أعلى مستوى من الرضا (kotler,2005:472). إن مستويات رضا الزبون سابقة الذكر تتأثر بالعديد من العوامل التي مني الترميا حددها كل من (3) (singh \& Harkiranpal, 2006, 2) الآتي:

4. عندما يكون الزبون راضياً عن الخدمات المقدمة فانهُ سيكرد عملية الشراء مما يسهل الاحتفاظ به. 5. يعد رضا الزبائن بمثابة التغذية العكسية للشركة بخصوص به الاهـ الخدمات المقدمة إليه، والتي تحدد المشاكل السائدة والمحتملة، مما يجعل الشركة تطور دائما الخدمات المقدمة للزبائن. 3.3. ولاء الزبون: لقد أعدت غالبية الشركات المسوقة للمنتجات برامج لتطوير ولاء الزبون كجزء من أنشطتها لبناء العلاقات مع الزبائن وتطويرها (88, (AL-Rubaiee \& AL-Nazer, 2010 ويشير (معلا، 2010، 55) الى أنَّ ولاء الزبون مو التزام عميق من جانب العميل باعادة شراء منتج مفضل لديهِ ورعايتهِ من خلال اتخاذ مواقف ايجابية نحوهُ والتحدث عنهُ بايجابية وقيامه بتوصية الآخرين بالشراء مع عدم وجود أي نوايا بالتحول الى اصناف أخرى في حالة تعرضه لأية حملة ترويجية تستهدف إثنائه عن التعامل مع الشركة الحالية والتحول الى شركة اخرى. وينفس السياق يشير (هواري وأمينة، 2012، 38) أنَّ ولاء الزبون هو موقف ايجابي للزبون نحو المنتج أو العلامة أو الشركة، يتضح في تكرار السلوك الشرائي. ويشير (الامين، 2009، 141) الى أنَّ الزبيون الذي يتميز بالولاء هو الزبون الذي يشتري نفس العلامة عدة مرات ويشكل متتالي، ومنه يمكن تحديد وقياس الولاء بطريقة عملية
1. توفر الرقابة الإستراتيجية الفعالة تغذية عكسية خاصة بمدى جودة أداء الشركة وأعضائها في الوقت المناسب. 2. تقدم الرقابة الإستراتيجية الفعالة وسائل تحفز الموظفين كثيراً للعمل من أجل تحقيق الاهداف الاستراتيجية للشركة.

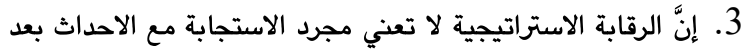
وقوعها، إذ أنّها تعني أيظاً وضع الشركة على الطريق الصحيح، واستباق الأحداث المتوقعة، والاستجابة السريعة للفرص والتهديدات

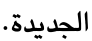

\section{3.المبحث الثاني}

\section{3 التفوق التسويقي:}

تسعى الشركات لمواكبة التطور في شتى المجالات كالتطور التقني، والتطور والتغير في أذواق المستهلكين، ولذلك ينبغي عليها إتباع استراتيجيات تسويقية ملائمة من أجل الوصول الى تحقيق تفوق

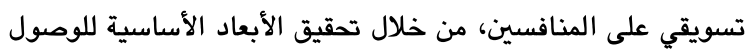
الى التفوق التسويقي، والتي تتمثل في الأبعاد الآتية 2.3. رضا الزبون:

يشكل رضا الزيون معياراً أساسياً للأداء التسويقي، ومكوناً جوهرياً لأساليب قياس رضا الزيون، وريما لا يوجد مقياس أداء الأعمال

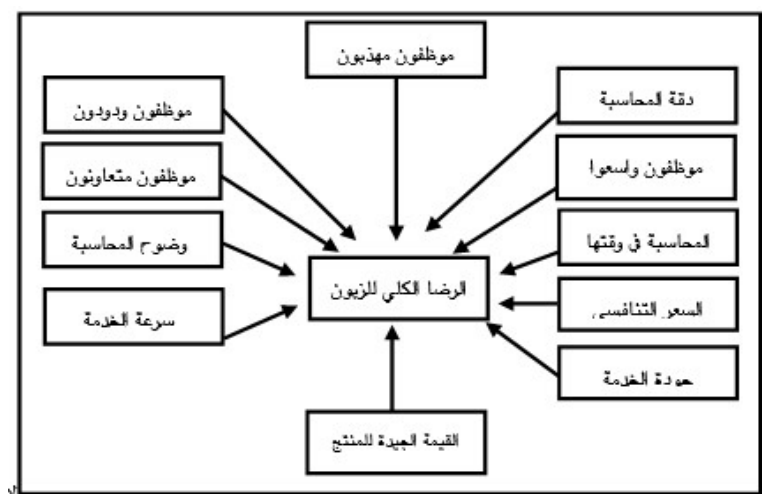

شكل (3):العوامل المؤثرة على رضا الزبون

Source: (Singh \& Harkiranpal, 2006)

وتبرز أممية رضا الزبائن لكونها أحد أهم العوامل التي تمكن الشركات من البقاء والاستمرار في السوق، ويالخصوص إذا وضعت رضا الزبائن كهدف من أهدافها الإستراتيجية اعترافاً بالدود الذي يؤديه رضا الزبون على مستقبل الشركة. ويمكن إبراز النقاط الآتية التي تصدد أهمية رضا الزبون: (نسيمة، 2011، 81، 8وني 1. تجنب المؤسسات الضغوط التنافسية ويخاصة المنافسة السعرية 2. إن رضا الزيائن عن الخدمات المقدمة يقلل من احتمالية توجه الزيائن إلى الشركات المنافسة. 3. إن الزبون الراضي عن إدارة الشركة وموظفيها بسبب استجابتهم لاحتياجاته والتميز في تقديم الخدمات يصبح أداة لجذب زبائن جدد. 
• • إن أفضل الطرق للوصول إلى ولاء الزبائن هي تجاوذ توقعاتهم.

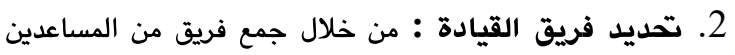
ممن يملكون مهارات التعامل مع الزبائن، وتوجه إليهم مهمة قيادة جهود بناء الولاء، ومنحهم الموارد الضرورية لذلك، ويجب اختيار أشخاص من المؤهلات المختلفة في المؤسسة، ومن المستويات المختلفة، والذين تتوفر فيهم المؤهلات التالية: موقف متميز تجاه

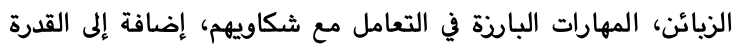
على الإبداع، والحماس، وغيرها من المؤهلات المميزة.

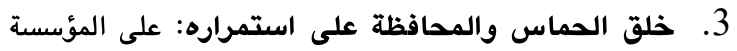

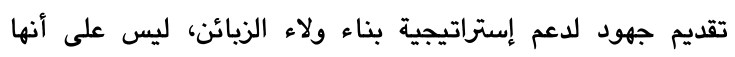

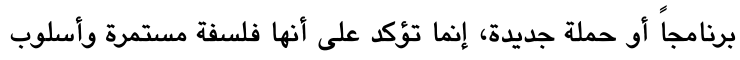
يجب أن تتبعةُ المؤسسة على المدى الطويل.

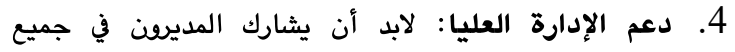

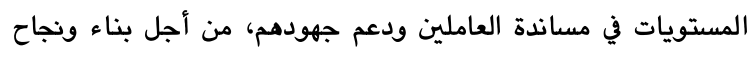
ولاء الزبون للمنظمة. 5. التدريب والتحفيز: يحتاج بناء ولاء الزبون إلى إقامة دورات لإنات تدريبية لتوفير المعلومات والمهارات لخلق الفرص ومساعدة العاملين على فهم كيفية استفادتهم من الموقف، وخلق الحماس والالتزام الضرودي من أجل النجاح المستمر. 4.3. قيمة الزبون: قيمة الزيون هو تفضيل العميل المتصور وتقييم تلك المنتجات

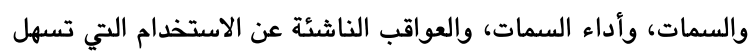
(أو تعوق) تحقيق أهداف الزيون وأغراضِه في حالات الاستخدام.

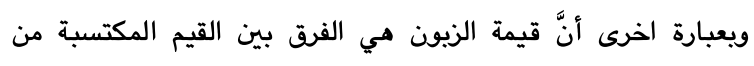

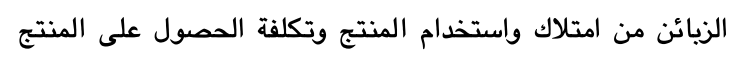
(Yamamoto, 2014, 549)

ومن وجهة نظر (قحف، 2015، 10) فيرى أن قيمة الزبون هي حكم

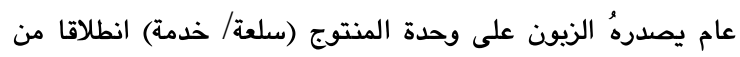

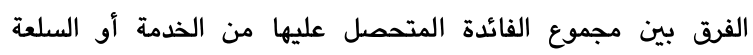

$$
\text { ومجموع التكاليف التي قدمها للحصول عليه. }
$$

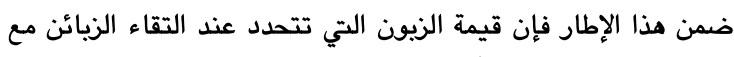

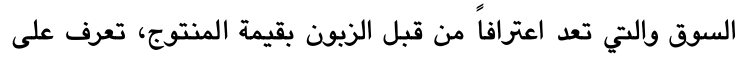

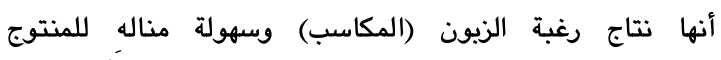

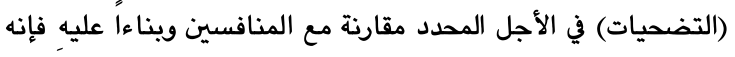

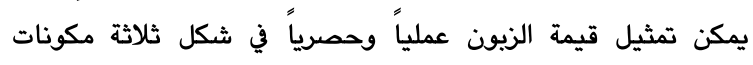

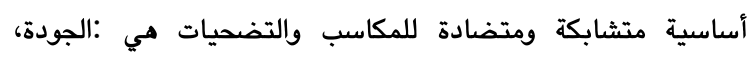
التكاليف، والأجل (خنشور، 2006، 380 ـ 381).

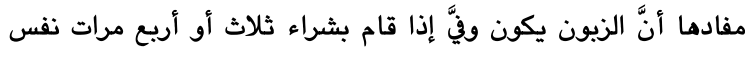

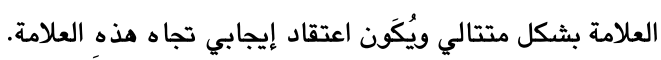

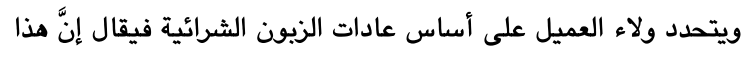

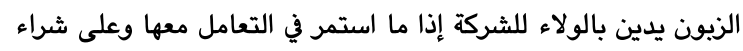

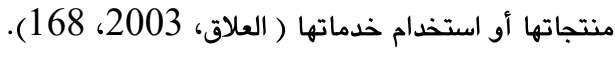

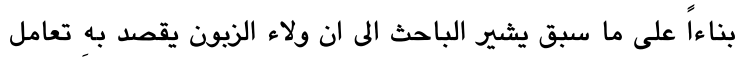

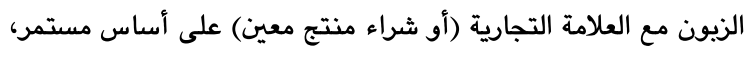
هو مقياس نجاح الشركة في الاحتفاظ بعلاقة طويلة الأمد مع الزبون. إن زيادة الولاء يمكن أن يحقق وفورات في التكاليف لشركة في ستة

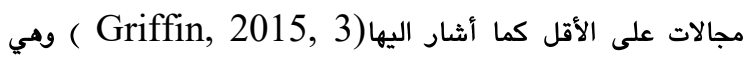

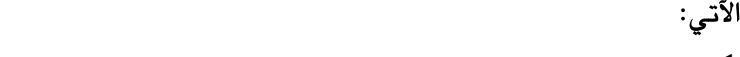
1. انخفاض تكاليف التسويق (لان تكاليف اكتساب عملاء جدد تتطلب المزيد من التكاليف) 2. انخفاض تكاليف المعاملات مثل التفاوض على العقود ومعالجة

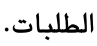
3. انخفاض نفقات دودان العملاء (عدد أقل من العملاء المفقودين).

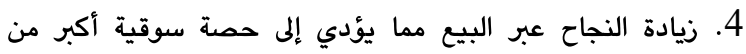

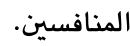
5. خفض تكاليف التنسيق والاتصالات.

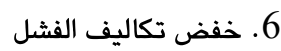

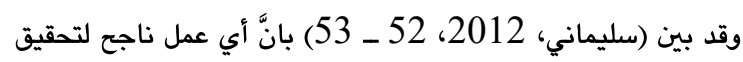

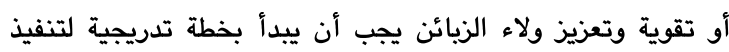
إستراتيجية ولاء الزبائن، وكالآتي:

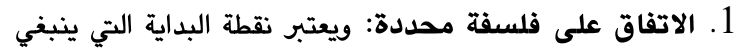

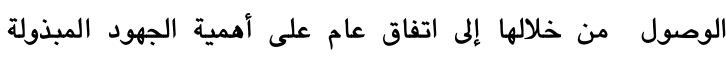

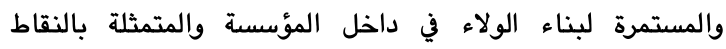
الأساسية التالية:

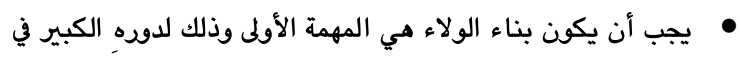

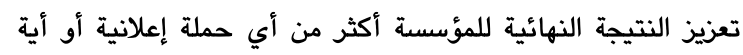
جهود للعلاقات العامة ...إلخ.

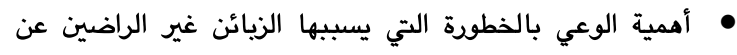

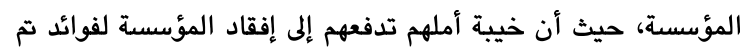

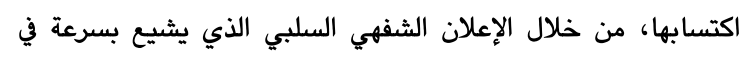

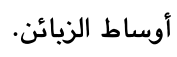
• أن كسب زبون جديد يكلف على الأقل خمس مرات ما يكلفهُ زبون حالي موجود. • إن علاقات العملاء الداخليين تعتبر جزءاً أساسيا حاسماً في عملية بناء ولاء الزبائن. 
بهدف بيان العلاقة والأثر بين الادارة الاستراتيجية في تحقيق التفوق التسويقي، يستلزم الأمر التحقق من صحة الفرضيات والتي تؤكد على وجود علاقة إرتباط وتاثير معنوي بين الادارة الاستراتيجية في تحقيق التفوق التسويقي في الشركات المبحوثة، وقبل الدخول في تحديد العلاقة والأثر بين متفيرات البحث، نقدم نبذة عن عينة البحث. 1.5.3. وصف عينة البحث: يعرض هذا المحور وصفاً لعينة

$$
\text { 1. البحث وكالآتي: وصف الشركات المبحوثة }
$$

تعد الشركات الصناعية المبحوثة في محافظة دهوك البوادر الاولى لتشكيل وتاسيس شركات صناعية كبيرة ذات رؤية استراتجية واضحة تساهم في تلبية احتياجات الاسوق المحلية في كردستان العراق بالمنتجات الضرورية، وتقلل من الإعتماد على المنتجات المستوردة من خارج الاقليم، مما يساهم في تعزيز مسيرة التنمية الاقتصادية والمساهمة بشكل فعال في دعيم وتنشيط عجلة الإقتصاد .وتتمثل عينة الدراسة بخمس شركات صناعية والعاملة في محافظة دموك، كما في

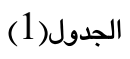

ويمكن الاشارة من خلال المخطط (4) الى آلية الشركة في تحقيق تفوق تسويقي على المنافسين متمثلة بـ (تحقيق زيادة المبيعات، وتحقيق مستويات ريح عالية) من خلال تفعيل وتعزيز الأبعاد الثلاثة المكونة للتفوق التسويقي.

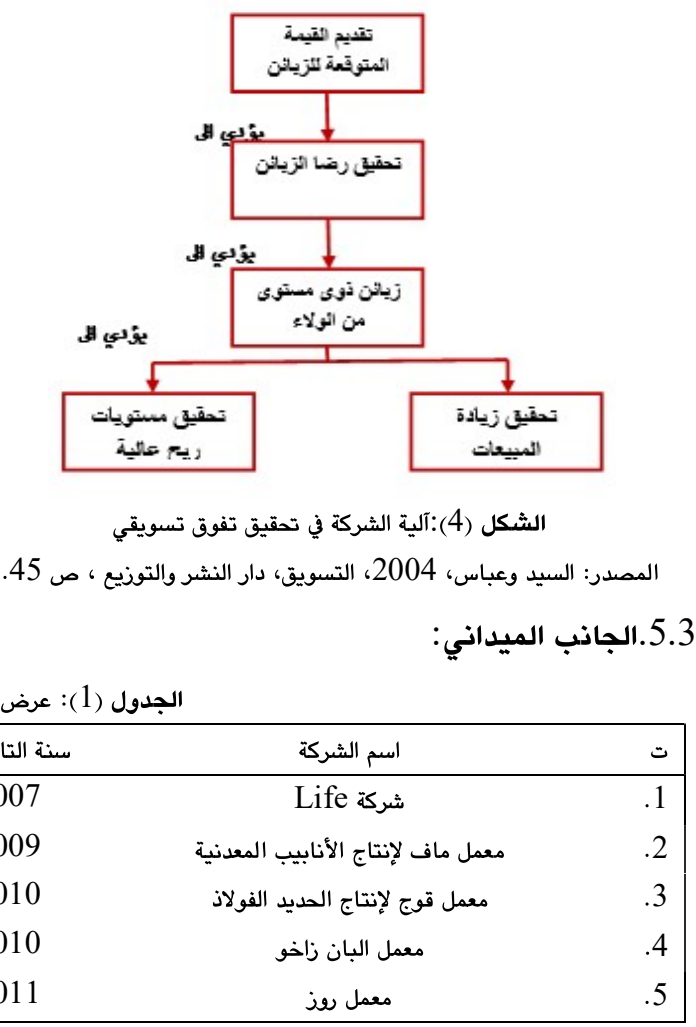

المصدر: من إعداد الباحث

2. وصف الافراد المبحوثين

جاءت محاولة الباحث لتناول موضوع دور الادارة الاستراتيجية في فرضياتها بواسطة مجموعة من الأساليب الإحصائية ومن خلال

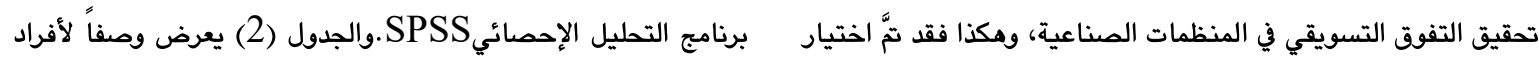
الكوادر في مذه الشركات وضمن المستويات التنظيمية المختلفة، إذ عينة البحث بحسب المركز الوظيفي والتحصيل الدراسي ومدة الخدمة

وزعت 40 استمارة استبانة على الكادر الوظيفي في خمس شركات للمبحوثين في الشركات المبحوثة. صناعية عينة البحث. تم من خلالها جمع بيانات البحث، وتم إختبار الجدول (2): وصف الأفراد المبحوثين في الشركات المبحوثة وتئة

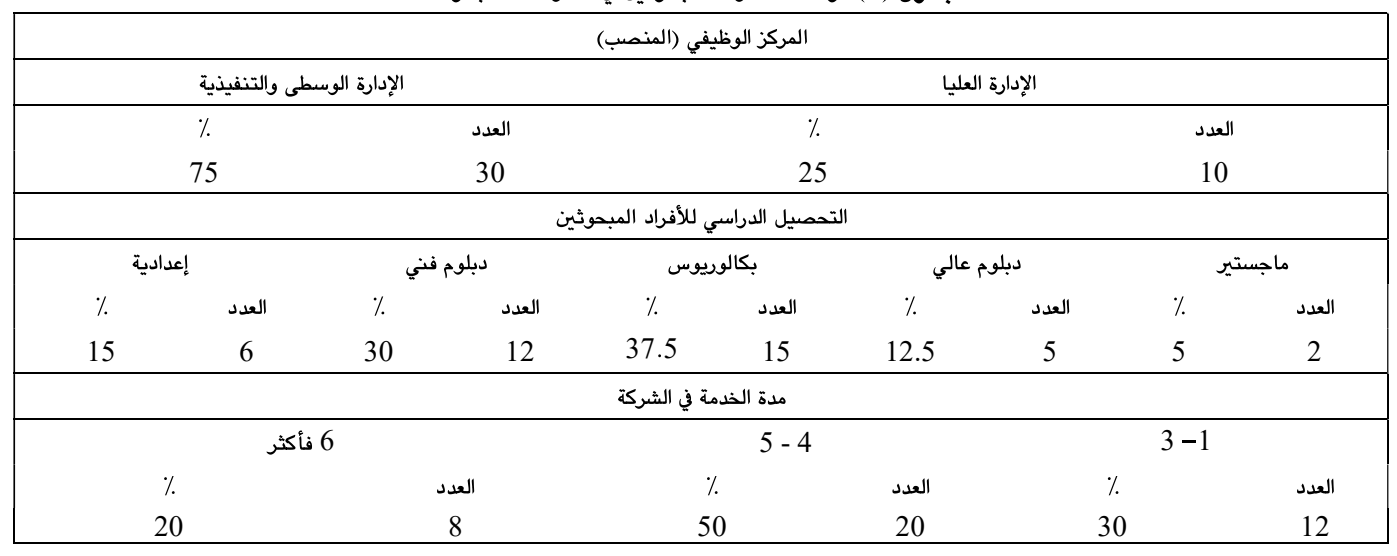


1. علاقات الإرتباط بين الادارة الاستراتيجية وتحقيق التفوق

$$
\text { التسويقي }
$$

يشير الجدول (3) الى نتائج علاقة الإرتباط بين متغيرات البحث.
2.5.3. اختبار نموذج البحث وفرضياته

بهدف التعرف على طبيعة العلاقة والأثر بين دود الادارة الاستراتيجية

في تدقيق التفوق التسويقي في الشركات الصناعية، خصص مذا المحور للتحقق من مدى سريان نموذج البحث وكالآتي:

\begin{tabular}{|c|c|c|c|c|}
\hline \multicolumn{2}{|c|}{ المؤشر الكلي } & \multicolumn{2}{|c|}{ التفوق التسويقي } & \multirow[t]{2}{*}{ المتفير المعتمد } \\
\hline & قيمة الزبون & ولاء الزبون & رضا الزيون & \\
\hline $0.730 *$ & $0.650^{*}$ & $0.763^{*}$ & $0.710^{*}$ & الادارة الاستراتيجية \\
\hline
\end{tabular}

$$
\mathrm{N}=0.0540 * \mathrm{p} \leq 0.05
$$

معنوية عند مستوى

المصدر: إعداد الباحث بالاعتماد على نتائج الحاسبة الالكتونية عندية

وتبين من الجدول (4) أن أقوى العلاقات كانت بين الادارة الاستراتيجية وولاء الزبون بدرجة ارتباط (0.763 )، وتليها كل من رضا الزيون وقيمة الزبون بدرجات ارتباط (0.710) ، (0.650)

$$
\text { على التوالي. }
$$

تأسيساً على ما تقدم تقبل الفرضية الفرعية للبحث والتي تنص على وجود علاقة إرتباط بين الإدارة الإستراتيجية مع كل بُعد من أبعاد

$$
\text { التفوق التسويقي في الشركات المبحوثة. }
$$

2. علاقة التاثير بين الإدارة الإستراتيجية والتفوق التسويقي على

$$
\text { مستوى الشركات المبحوثة. }
$$

يعرض الجدول (4) نتائج علاقة التاثير بين الإدارة الإستراتيجية

$$
\text { والتفوق التسويقي. }
$$

يبين الجدول (4) نتائج علاقة الإرتباط بين الادارة الاستراتيجية والتفوق التسويقي في الشركات المبحوثة، إذ نلاحظ وجود علاقة إرتباط معنوية موجبة بين المتغير المستقل (الادارة الاستراتيجية)، والمتغير المعتمد (التفوق التسويقي). ويلغت قيمة معامل الإرتباط للمؤشر الكلي (0.730) وهي معنوية عند مستوى (0.05) ومو دليل على قوة العلاقة بين متغيرات البحث. تأسيساً على ما تقدم تقبل الفرضية الرئيسة للبحث والتي تنص على وجود علاقة ارتباط معنوية موجبة بين الإدارة الإستراتيجية وتحقيق التفوق التسويقي في

\begin{tabular}{|c|c|c|c|c|c|}
\hline \multicolumn{2}{|c|}{$\mathrm{F}$} & \multirow[b]{2}{*}{$\mathrm{R}^{2}$} & \multicolumn{2}{|c|}{ الادارة الاستراتيجية } & \multirow{2}{*}{ المتغير المستقل } \\
\hline الجدولية & المحسوية & & $\mathrm{B}_{1}$ & $\mathrm{~B}_{0}$ & \\
\hline 4.07 & 82.65 & 0.53 & $\begin{array}{l}0.755 \\
(8.23)\end{array}$ & 0.845 & التفوق التسويقي \\
\hline
\end{tabular}
الشركات المبحوثة. ومن أجل توضيح علاقة الإرتباط على المستوى الجزئي بين الادارة الاستراتيجية مع كل بعد من أبعاد التفوق التسويقي في الشركات المبحوثة، تم اختبار الفرضيات الفرعية المنبثقة عن الفرضية الرئيسة، الجدول ( 4 ): تاثير الإدارة الإستراتيجية في تحقيق التفوق التسويقي على مستوى الشركات المبحوثة

الجدول من إعداد الباحث بالاعتماد على نتائج الحاسبة الالكتونية N

$$
\text { df }(1,39) \text { (8.23) }
$$

(8.23) وهي قيمة معنوية وأكبر من قيمتها الجدولية والبالغة تشير نتائج الانحدار على مستوى هذهِ الشركات إلى وجود تأثير (1.684) عند مستوى معنوي (0.05). معنوي موجب بين الادارة الاستراتيجية والتفوق التسويقي، إذ بلغت قيمة (F) المحسوية ( 82.65 ) وهي أعلى من قيمتها الجدولية ويهدف توضيع علاقة الأثر بين الادارة الاستراتيجية مع كل بعد من

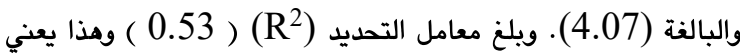
أبعاد التفوق التسويقي على مستوى الشركات المبحوثة، فقدتَّ تحليل علاقات الأثر بين الإدارة الإستراتيجية مع كل بعد من أبعاد التفوق التسويقي على مستوى الشركات المبحوثة بصورة منفردة كما في أن (53 \% \% من الاختلافات المفسرة في الادارة الاستراتيجية تفسرها التفوق التسويقي، ويعود الباقي الى متغيرات عشوائية لا يمكن السيطرة عليها أو إنها غير داخلة في نموذج الانحدار أصلاً. ومن خلال متابعة معاملات (B) واختبار (t) لها تبين أن قيمة (t) المحسوية 
الجرجري، خضير خليل شيخو / مجلة العلوم الانسانية لجامعة زاخو، مجلد:6 ، العدد: 1 ، الجزء:2،ص 280- 289، آذار 2018.

\begin{tabular}{|c|c|c|c|c|c|c|c|c|}
\hline & & $\mathrm{F}$ & $\mathrm{R}$ & قيمة الزبون & ولاء الزيون & رضا الزيون & & المتغير المعتمدة المتغيرات \\
\hline & الجدولية & المسسوبة & & B3 & B2 & B1 & Bo & المستقل \\
\hline 2.86 & 4.5 & & 0.8 & $\begin{array}{l}0.24 \\
*(7.15)\end{array}$ & $\begin{array}{l}0.265 \\
(6.4)^{*}\end{array}$ & $\begin{array}{l}0.25 \\
*(7.65)\end{array}$ & 1.7 & الادارة الاستراتيجية \\
\hline
\end{tabular}

3. تبين من خلال الدراسة إنَّ تطبيق الإدارة الإستراتيجية لهُ اثر

إيجابي على كفاءة وفعالية التفوق التسويقي في الشركات المبحوثة .

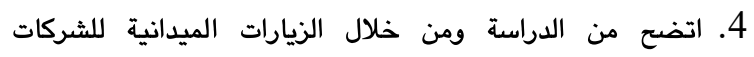

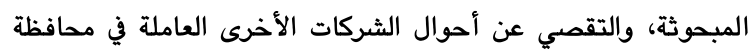

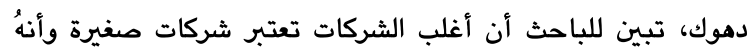

ولحد الآن لم يشهد تاسيس الشركات الكبيرة حسب علم الباحث.

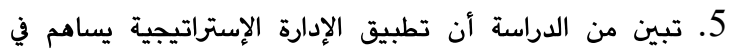

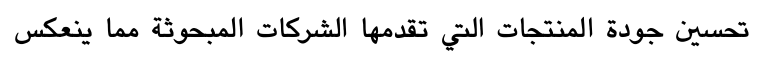

$$
\begin{aligned}
& \text { ايجاباً على تحقيق التفوق التسويقي. } \\
& \text { 2.6.المقترحات: }
\end{aligned}
$$

بناءاً على الإستنتاجات التي توصل اليها الباحث، يرى الباحث

$$
\text { ضرورة تقديم المقترحات الآتية: }
$$

1. أظهرت نتائج الزيارات الميدانية للشركات المبحوثة أنية: أن هناك

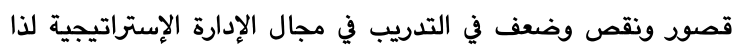
لابد من السعي لزيادة وتكثيف الدورات التدريبية في مجال الإدارة

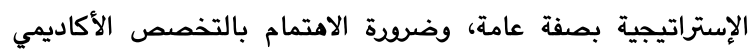

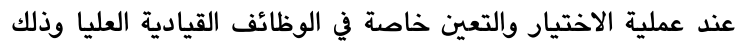
لانَّ للتخصص أثر كبير على شاغل الوظيفة.

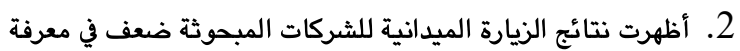

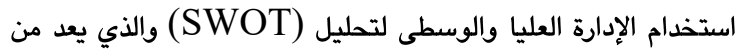

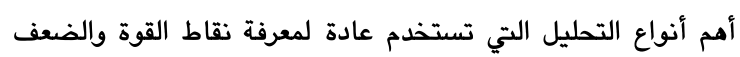
والفرص والتهديدات في البيئة الداخلية والخارجية، وذلك لإستثمارها في تحقيق التفوق التسويقي، لذا يرى الباحث ضرودة صرف المزيد من الجهد لرفع مستوى معرفة وتطبيق أساليب التحليل الاستراتيجي للإدارة العليا والوسطي في هذه الشركات. 3. يرى الباحث ضرودة إقامة الشركات المتوسطة والكبيرة في اقليم كرستان العراق، وللقطاعين العام والخاص، ويستلزم ذلك توفير الدعم

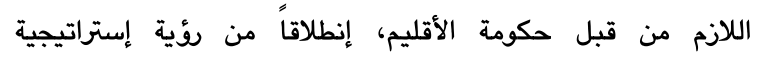

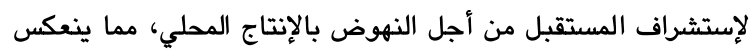

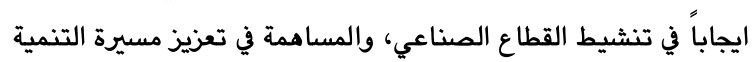
الاقتصادية، وتحقيق تفوق تسويقي لمنتجاتها.
تشير نتائج الاندار على مستوى الشركات المبحوثة إلى وجود تأثير معنوي موجب لدود الإدارة الاستراتيجية مع كل بعد من أبعاد التفوق

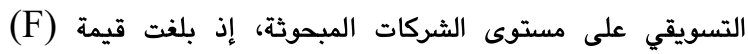

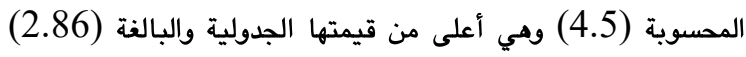

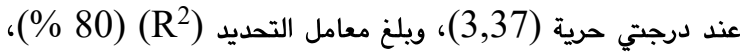

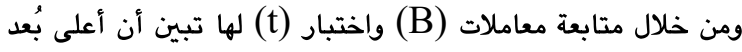

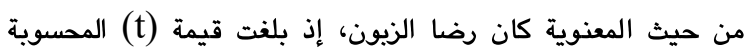

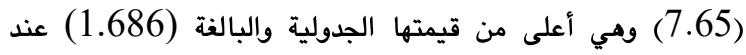

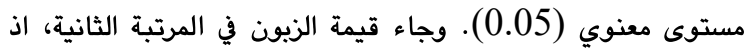
بلغت قيمة (t) المحسوية (7.15) وهي أعلى من قيمتها الجدولية

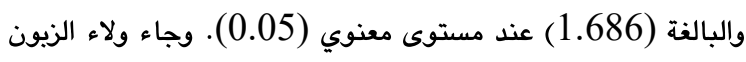

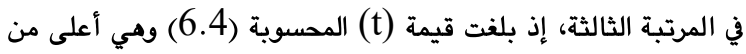
قيمتها الجدولية والبالغة (1.686) عند مستوى معنوي (0.05). تاسيساً على ما تقدم تقبل الفرضية التي تنص على وجود علاقة معنوية موجبة بين الادارة الإستراتيجية مع كل بعد من أبعاد التفوق التسويقي في الشركات المبحوثة. 6.الاستنتاجات والمقترحات

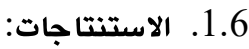
توصل الباحث من خلال أدبيات الدراسة، وتحليل بيانات الدراسة

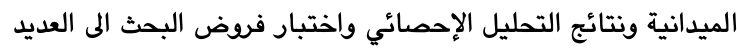

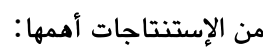
1. اظهر التحليل الإحصائي والاختبارات والمقاييس الإحصائية التي استخدمت في هذه الدراسة إثبات صحة جميع فرضيات البحث.

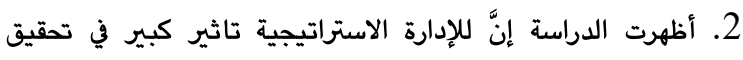
أبعاد التفوق التسويقي والمتمثلة بـ (رضا الزيون، وولاء الزيون،

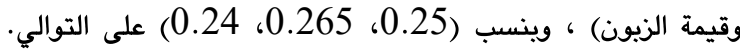

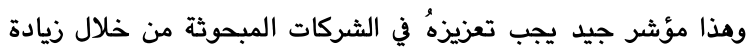

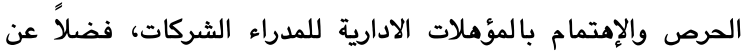
الاهتمام بالدورات التدريبية المتخصصة وبرامج الدراسات العليا لزيادة وعي المدراء حول مفاهيم وأساليب الإدارة الإستراتيجية. 


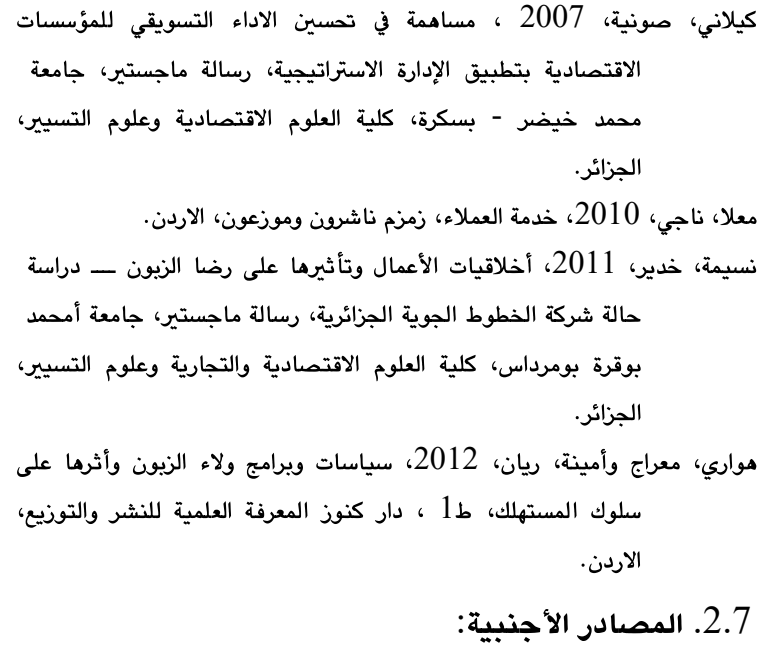

AL-Rubaiee \& AL-Nazer, 2010, investigate the impact of relationship marketing orientation on customer loyalty.

Bowen, J.T. and chen, S. 2001, "The relationship between customor loxalty" and customer salis faction", in ternational Journal of contemporary Hospitality management, vol 5 Nos $4 / 5$.

David, Fred R., 2011, Strategic Management concepts and cases, by Pearson Education, Inc., publishing as Prentice Hall, One Lake Street, Upper Saddle River, New Jersey 07458. Library of Congress, United States of America.

Griffin, Jill, 2015, Customer Loyalty, https://altfeldinc.com/pdfs.

Kotler, Philips "Marketing Management", France: Pearson education, 12 èmeédition 2006, P 172.

Kotler, Philips, 2005, principles of marketing, Eleventh Edition, prentice hall, New Delhi, India.

Nedelea, Stefan ,2009, The Importance of the Strategic Management Process in the Knowledge-Based Economy, The Bucharest Academy of Economic Studies, Romania, Volume 10, Issue 1, March 2009.

Sepehri, mehran \& Kermanshah, Ali, 2012, strategic planning, graduate school of management. U. S. A.

Singh, Harkianpal, 2006, the Importance of customer satisfaction in Relation to Gustomer Loyalty and Retention Asia Pacific university college of Te chu-ologys Innovation, Tecnaology Park malasia, Vet I working Paper wp- 06-06.

White, Colin, 2004, Strategic Management, Library of Congress, U. S. A.

Yamamoto, gonca telli, 2014, Understandind customer value concept: key to success, Maltepe University, Faculty of Economics and Administrative Sciences, Business Department, Sahilyolu Orhantepe Mah. Yakamoz

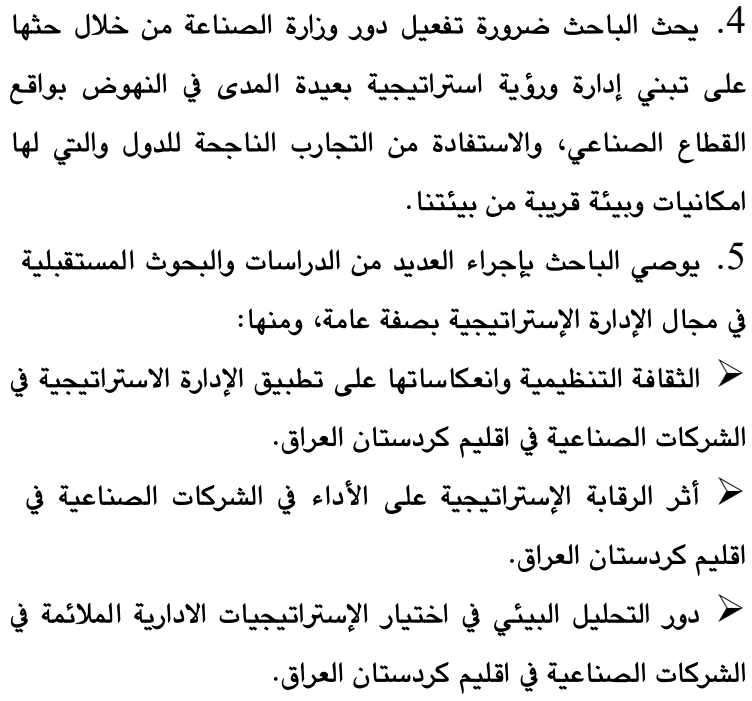

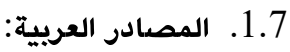

الامين، عبد الحفيظي محمد، 2009، دور ادارة التسويق في كسب الزبون - دراسة

حالة مؤسسة مولاي للمشروبات الغازية، رسالة ماجستير، كلية العلوم

$$
\text { الاقتصادية والتسير، الجزائر. }
$$

تبيدي، محمد حنفي محمد نور، 2010، أثر الإدارة الإستراتيجية على كفاءة وفعالية الأداء، اطروحة دكتوراة، جامعة النيلين، السودان.

خنشور، جمال، أثر القيمة - الزبون والمفاهيم المحاذية لها على مردودية المؤسسة،

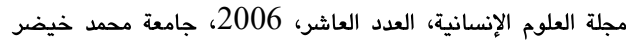

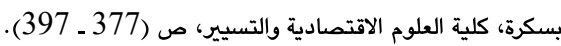

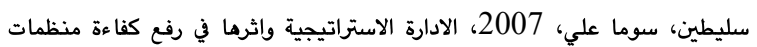
الاعمال، رسالة ماجستير، كلية الاقتصاد، جامعة تشرين، الجمهورية

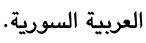

السلىماني، حنان بنت عبد الرحمن، 2012، رؤىة مستقبلى لتطبـى لتقى الإدارة

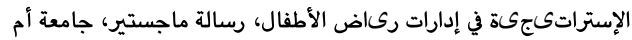

$$
\text { السيد، أحمد وعباس، نبيلة، 2004، القرى، التسويق، دار النشر والتورية، السعودية. }
$$

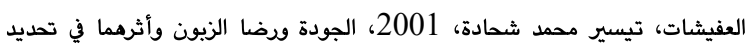
الاستراتيجيات التسويقية للخدمات المصرفية. دراسة لاراء عينة في

المصارف التجارية الاردنية، اطرحة دكتوراه غير منشورة، كلية الادارة

$$
\text { والاقتصاد، جامعة الموصل. }
$$

العلاق، بشير، 2003، تطبيقات على التسويق بالعلاقات، دار زهران للنشر

$$
\text { والتوزيع، عمان. }
$$

قحف، هالة، 2015، دود التسويق بالعلاقات في تعزيز ولاء الزبائن للعلامة موبيليس

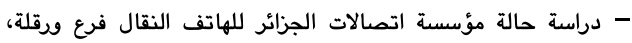

جامعة قاصدي مرباح- ورقلة كلية العلوم الاقتصادية والتجارية وعلوم

$$
\text { التسيير قسم العلوم التجارية، الجزائر. }
$$




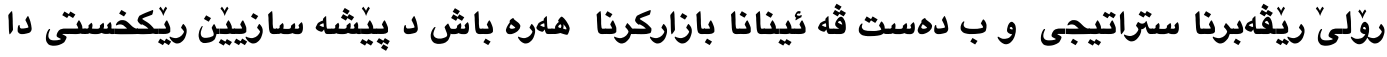

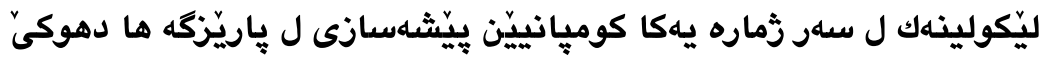

ئه ثروكه كوميانييين بِيشهانى درينكه مه كا بزه حمه تردا كار دكهن ، زيهر كو تيكه ل بوونا بازاريت جيهانى ويه يدا بوونا بازاريت نوى وسيسته مين

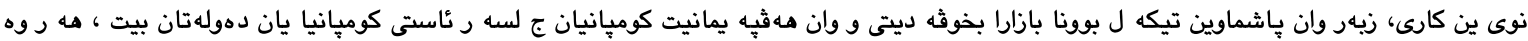

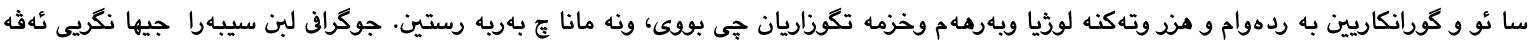

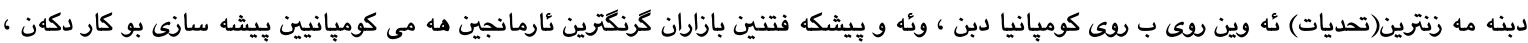

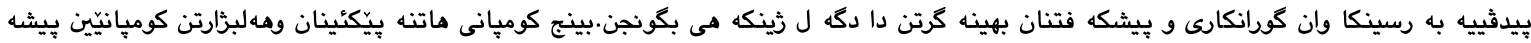

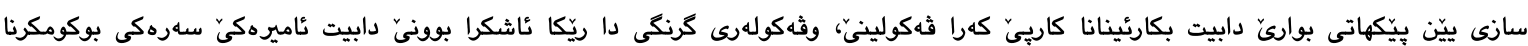

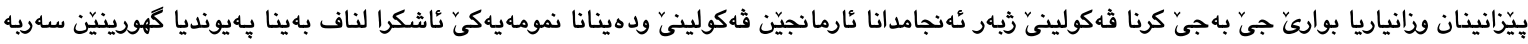

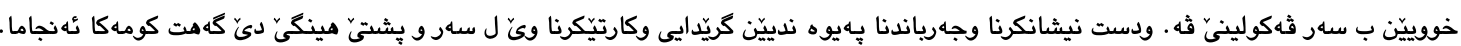

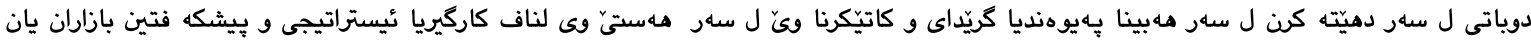

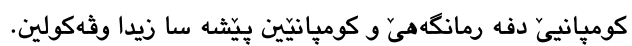

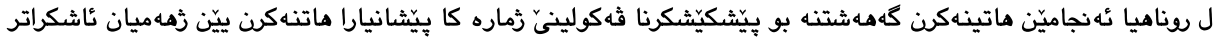

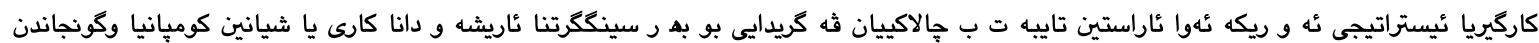
دكه ل كورانكاريين نافخوى وده رفه.

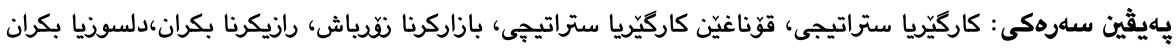

\section{The role of strategic management in achieving marketing excellence in industrial organization "an exploratory study on a number of industrial companies in dohuk governorate"}

\begin{abstract}
:
Today, industrial companies are operating in a more complex environment because of the integration of international markets and the emergence of new markets and new rules of labor because of mergers and alliances of companies whether at the level of companies or countries. The constant change in ideas, technology, tastes, products and services and the disappearance of geographical divisions in the face of globalization are considered One of the biggest challenges faced by companies, and since the marketing excellence of the most important goals pursued by all industrial companies, it is necessary to face change and keep pace with progress and adaptation to the environment. The strategic management is the way to determine the directions of activities to address the problems and employ the possibilities of the company and adapt to internal and external changes .A group of five industrial companies was chosen as a field for field research. The researcher adopted the questionnaire method as a main tool for collecting data and information in the practical aspect of the research. In order to achieve the research objectives and hypotheses, based on the description of the research variables and their diagnosis and the test of correlation and impact relationships, a number of conclusions were reached which confirmed the existence of a correlation relationship and a significant effect between the strategic management and the marketing superiority of the investigated companies. In light of the conclusions reached by the researcher a number of proposals, most notably the need to activate the role of senior management in the adoption of strategic management to enhance the achievement of marketing excellence of companies surveyed.
\end{abstract}

keywords: Strategic management, strategic management components, marketing excellence, customer satisfaction, customer loyalty. 